\title{
ON SOME CLASSES OF SPIRALLIKE FUNCTIONS DEFINED BY THE SALAGEAN OPERATOR
}

\author{
MOHAMMAD MEHDI SHABANI ${ }^{1}$, SAEED HASHEMI SABABE ${ }^{2 *}$ \\ 1 Department of Mathematics, University of Shahrood, Shahrood, Iran. \\ 2 Young Researchers and Elite Club, Malard Branch, Islamic Azad University, \\ Malard, Iran. \\ ORCID ID: 0000-0003-1416-7795 ${ }^{1}$, 0000-0003-1167-5006 ${ }^{2}$
}

\begin{abstract}
In this paper, we introduce two subclasses of analytic and Spirallike functions and investigate convolution properties, the necessary and sufficient condition, coefficient estimates and inclusion properties for these classes.
\end{abstract}

\section{PREFACE}

In recent times, the study of analytic functions has been useful in solving many problems in mechanics, Laplace equation, electrostatics, etc. An analytic function is said to be univalent in a domain if it does not take the same value twice in that domain. Let us denote the family of all meromorphic functions $f$ with no poles in the unit disk $\mathbb{U}:=\{z \in \mathbb{C}:|z|<1\}$ of the form

$$
f(z)=z+a_{2} z^{2}+a_{3} z^{3}+\cdots=z+\sum_{k=2}^{\infty} a_{k} z^{k},
$$

by $\mathcal{A}$. Clearly, functions in $\mathcal{A}$ are analytic in $\mathbb{U}$ and the set of all univalent functions $f \in \mathcal{A}$ is denoted by $\mathcal{S}$. Functions in $\mathcal{S}$ are of interest because they appear in the Riemann mapping theorem and several other situation in many different contexts. In 1983, Salagean [?] introduced differential operator $\mathcal{D}^{k}: \mathcal{A} \rightarrow \mathcal{A}$ defined by

$$
\begin{gathered}
\mathcal{D}^{0} f(z)=f(z), \\
\mathcal{D}^{1} f(z)=\mathcal{D} f(z)=z f^{\prime}(z), \\
\mathcal{D}^{n} f(z)=\mathcal{D}\left(\mathcal{D}^{n-1} f(z)\right)=z\left(\mathcal{D}^{n-1} f(z)\right)^{\prime}, \quad n \in \mathbb{N}=\{1,2,3, \ldots\} .
\end{gathered}
$$

In this way

$$
\mathcal{D}^{n} f(z)=z+\sum_{k=2}^{\infty} k^{n} a_{k} z^{k}, \quad n \in \mathbb{N}_{0}=\{0\} \cup \mathbb{N} .
$$

For functions $f$ given by $(1)$ and $g$ given by

$$
g(z)=z+b_{2} z^{2}+b_{3} z^{3}+\cdots=z+\sum_{k=2}^{\infty} b_{k} z^{k},
$$

E-mail address: Mohammadmehdishabani@ymail.com, Hashemi_1365@yahoo.com.

2010 Mathematics Subject Classification. 30C45; 30C50.

Key words and phrases. Analytic function, Hadamard product, Starlike function, Convex function, Subordination, Salagean differential operator.

* Corresponding Author. 
the Hadamard product or convolution of $f(z)$ and $g(z)$ is defined by

$$
f(z) * g(z)=z+\sum_{k=2}^{\infty} a_{k} b_{k} z^{k}
$$

In this paper, we investigate convolution properties of $\mathcal{S}_{n}^{\alpha}[A, B]$ and $\mathcal{K}_{n}^{\alpha}[A, B]$ associated with Salagean differential operator. Using convolution properties, we find the necessary and sufficient condition, coefficient estimates and inclusion properties for these classes. More recent works can be found on $[2,5,10,12,16]$.

\section{Preliminaries}

We start with some useful definitions, theorems and lemmas.

Definition 2.1. A function $f \in \mathcal{S}$ is said to be starlike in $\mathbb{U}$ if the image $f(\mathbb{U})$ is starlike with respect to 0 . It is well known that a function $f$ is starlike if and only if

$$
\operatorname{Re}\left(\frac{z f^{\prime}(z)}{f(z)}\right)>0 \quad(z \in \mathbb{U}) .
$$

We denote by $\mathcal{S}^{*}$ the class of all functions in $\mathcal{S}$ which are starlike in $\mathbb{U}$. A function $f \in \mathcal{S}$ is said to be convex in $\mathbb{U}$ if the image $f(\mathbb{U})$ is convex.

Lemma 2.2. The function $f$ is convex in $\mathbb{U}$ if and only if

$$
\operatorname{Re}\left(1+\frac{z f^{\prime \prime}(z)}{f^{\prime}(z)}\right)>0 \quad(z \in \mathbb{U}) .
$$

We denote by $\mathcal{K}$ the class of all functions in $\mathcal{S}$ which are convex in $\mathbb{U}$. It is easy to see that, $\mathcal{K} \subset \mathcal{S}^{*} \subset \mathcal{S} \subset \mathcal{A}$.

Definition 2.3. For analytic functions $g$ and $h$ in $\mathbb{U}, g$ is said to be subordinate to $h$ if there exists an analytic function $w$ such that

$$
w(0)=0, \quad|w(z)|<1, \quad \text { and } \quad g(z)=h(w(z)), \quad z \in \mathbb{U} .
$$

This subordination will be denoted here by

$$
g \prec h,
$$

or, conventionally, by

$$
g(z) \prec h(z),
$$

In particular, when $h$ is univalent in $\mathbb{U}$,

$$
g \prec h \Longleftrightarrow g(0)=h(0), \quad \text { and } \quad g(\mathbb{U}) \subset h(\mathbb{U}), \quad z \in \mathbb{U} .
$$

Making use of the principal of subordination and Salagean differential operator between analytic functions, we introduce the following classes of analytic functions for $n \in \mathbb{N}_{0},|\alpha|<\frac{\pi}{2}$ and $-1 \leq B<A \leq 1$ :

$$
\mathcal{S}_{n}^{\alpha}[A, B]=\left\{f \in \mathcal{S}: e^{i \alpha} \frac{z\left(\mathcal{D}^{n} f(z)\right)^{\prime}}{\mathcal{D}^{n} f(z)} \prec \cos \alpha\left(\frac{1+A z}{1+B z}\right)+i \sin \alpha, z \in \mathbb{U}\right\},
$$

and

$$
\mathcal{K}_{n}^{\alpha}[A, B]=\left\{f \in \mathcal{S}: e^{i \alpha}\left(1+\frac{z\left(\mathcal{D}^{n} f(z)\right)^{\prime \prime}}{\left(\mathcal{D}^{n} f(z)\right)^{\prime}}\right) \prec \cos \alpha\left(\frac{1+A z}{1+B z}\right)+i \sin \alpha, z \in \mathbb{U}\right\}
$$

Note that

$$
f(z) \in \mathcal{K}_{n}^{\alpha}[A, B] \quad \Longleftrightarrow \quad z f^{\prime}(z) \in \mathcal{S}_{n}^{\alpha}[A, B] .
$$

By specializing the parameters, we have the following know subclasses studied by various researhers. 
I $\mathcal{S}_{0}^{0}[A, B]=\mathcal{S}[A, B]$ and $\mathcal{K}_{0}^{0}[A, B]=\mathcal{K}[A, B]$, where the classes $\mathcal{S}[A, B]$ and $\mathcal{K}[A, B]$ are introduced and studied by many authors in $[1,6,8,9,14]$.

II $\mathcal{S}_{0}^{0}[1-2 \beta,-1]=\mathcal{S}^{*}(\beta)$ and $\mathcal{K}_{0}^{0}[1-2 \beta,-1]=\mathcal{K}(\beta)$, where the classes $\mathcal{S}^{*}(\beta)$ and $\mathcal{K}(\beta)$ are introduced and stadied in [7].

III $\mathcal{S}_{0}^{0}\left[\frac{b^{2}-a^{2}+b}{b}, \frac{1-b}{a}\right]=\mathcal{S}(a, b)$ and $\mathcal{K}_{0}^{0}\left[\frac{b^{2}-a^{2}+b}{b}, \frac{1-b}{a}\right]=\mathcal{K}(a, b)$, where the classes $\mathcal{S}(a, b), \mathcal{K}(a, b)$ are introduced and stadied by in $[14,15]$.

IV $\mathcal{S}_{0}^{\alpha}[A, B]=\mathcal{S}^{\alpha}[A, B]$ and $\mathcal{K}_{0}^{\alpha}[A, B]=\mathcal{K}^{\alpha}[A, B]=\mathcal{S}_{1}^{\alpha}[A, B]$, where the classes $\mathcal{S}^{\alpha}[A, B], \mathcal{K}^{\alpha}[A, B]$ are introduced and stadied in $[3,4,11]$.

$\mathrm{V} \mathcal{S}_{0}^{0}[A, B]=\mathcal{R}_{0}[A, B]$ and $\mathcal{K}_{0}^{0}[A, B]=\mathcal{R}_{1}[A, B]$, where the classes $\mathcal{R}_{0}[A, B], \mathcal{R}_{1}[A, B]$ are introduced and stadied in [1].

\section{Convolution Properties}

In this section, we study some of the properties of foresaid convolution. Unless otherwise mentioned, we assume throughout this paper that $-1 \leq B<A \leq 1$, $|\alpha|<\frac{\pi}{2},|\xi|=1$ and $\mathcal{D}^{n} f(z)$ is defined by (2). To prove our convolution properties, we shall need the following lemmas due to Silverman and Silvia [14].

Lemma 3.1. [14] The function $f(z)$ defined by (1) is in the class $\mathcal{S}^{*}[A, B]$ if and only if for all $z$ in $\mathbb{U}$ and all $\xi,|\xi|=1$,

$$
\frac{1}{z}\left[f * \frac{z+\frac{\xi-A}{A-B} z^{2}}{(1-z)^{2}}\right] \neq 0 .
$$

Lemma 3.2. The function $f(z)$ defined by (1) is in the class $\mathcal{S}_{n}^{\alpha}[A, B]$ if and only if for all $z$ in $\mathbb{U}$ and all $\xi,|\xi|=1$,

$$
\frac{1}{z}\left[f *\left(z+\sum_{k=2}^{\infty}\left(\frac{k-\psi}{1-\psi}\right) k^{n} z^{k}\right)\right] \neq 0
$$

where

$$
\psi=\frac{e^{i \alpha}+(A \cos \alpha+i B \sin \alpha) \zeta}{e^{i \alpha}(1+B \zeta)}
$$

Proof. An application of lemma 3.1 exhibits that $f \in \mathcal{S}_{n}^{\alpha}[A, B]$ if and only if

$$
\begin{aligned}
& e^{i \alpha} \frac{z\left(\mathcal{D}^{n} f(z)\right)^{\prime}}{\mathcal{D}^{n} f(z)} \neq \cos \alpha\left(\frac{1+A \zeta}{1+B \zeta}\right)+i \sin \alpha \quad(z \in \mathbb{U}, \quad|\xi|=1) \\
& \Longleftrightarrow z\left(\mathcal{D}^{n} f(z)\right)^{\prime}-\mathcal{D}^{n} f(z)\left(\frac{e^{i \alpha}+(A \cos \alpha+i B \sin \alpha) \zeta}{e^{i \alpha}(1+B \zeta)}\right) \neq 0 \quad z \in \mathbb{U},|\xi|=1 .
\end{aligned}
$$

Since $z f^{\prime}=f * \frac{z}{(1-z)^{2}}$ and $f=f * \frac{z}{1-z}$, we can write $\mathcal{D}^{n} f(z)=f(z) * h(z) * \frac{z}{1-z}$ and $z\left(\mathcal{D}^{n} f(z)\right)^{\prime}=f(z) * h(z) * \frac{z}{(1-z)^{2}}$, where $h(z)=z+\sum_{k=2}^{\infty} k_{n} z^{k}$ and Substituting $\psi:=\frac{e^{i \alpha}+(A \cos \alpha+i B \sin \alpha) \zeta}{e^{i \alpha}(1+B \zeta)}$, relation (8) is equivalent to

$$
f(z) * h(z) *\left(\frac{z}{(1-z)^{2}}-\frac{\psi z}{1-z}\right) \neq 0 \quad z \in \mathbb{U} .
$$

On the other hand, by extention $\frac{z}{(1-z)^{2}}$ and $\frac{z}{1-z}$, we have

$$
\frac{z}{(1-z)^{2}}-\frac{\psi z}{1-z}=z+\sum_{k=2}^{\infty}\left(\frac{k-\psi}{1-\psi}\right) z^{k}
$$

By substituting (10) in (9), the proof is complete. 
Theorem 3.3. A necessary and sufficient condition for the function $f$ defined by (1) to be in the class of $\mathcal{S}_{n}^{\alpha}[A, B]$ is that

$$
1-\sum_{k=2}^{\infty} \frac{(k-1)\left(e^{i \alpha}+i B \zeta \sin \alpha\right)-(A-k B) \zeta \cos \alpha}{(A-B) \zeta \cos \alpha} k^{n} a_{k} z^{k-1} \neq 0
$$

Proof. Notice that

$$
\frac{k-\psi}{1-\psi}=-\frac{(k-1)\left(e^{i \alpha}+i B \zeta \sin \alpha\right)-(A-k B) \zeta \cos \alpha}{(A-B) \zeta \cos \alpha},
$$

where $\psi$ was defined in (7). Using (12), we can write (6) as

$$
\frac{1}{z}\left[z-\sum_{k=2}^{\infty} \frac{(k-1)\left(e^{i \alpha}+i B \zeta \sin \alpha\right)-(A-k B) \zeta \cos \alpha}{(A-B) \zeta \cos \alpha} k^{n} a_{k} z^{k}\right] \neq 0
$$

Simplifying relation (13), we obtain (11) and the proof is complete.

Lemma 3.4. The function $f(z)$ defined by (1) is in the class of $\mathcal{K}_{n}^{\alpha}[A, B]$ if and only if for all $z$ in $\mathbb{U}$ and all $\xi,|\xi|=1$,

$$
\frac{1}{z}\left[f *\left(z+\sum_{k=2}^{\infty}\left(\frac{k-\psi}{1-\psi}\right) k^{n+1} z^{k}\right)\right] \neq 0
$$

where $\psi$ was defined in ( 7$)$.

Proof. Set

$$
g \prec h \quad \Longleftrightarrow \quad g(0)=h(0), \quad \text { and } \quad g(\mathbb{U}) \subset h(\mathbb{U}), \quad z \in \mathbb{U} .
$$

Note that

$$
z g^{\prime}(z)=z+\sum_{k=2}^{\infty}\left(\frac{k-\psi}{1-\psi}\right) k^{n+1} z^{k}
$$

From the identity $z f * g=f * z g^{\prime}$ and the fact that $f \in \mathcal{K}_{n}^{\alpha}[A, B]$ if and only if $z f^{\prime} \in \mathcal{S}_{n}^{\alpha}[A, B]$, from lemma 3.1 we have

$$
\frac{1}{z}\left[z f^{\prime}(z) * g(z)\right] \neq 0 \Longleftrightarrow \frac{1}{z}\left[f(z) * z g^{\prime}(z)\right] \neq 0
$$

By substituting relation (15) in (16), we have obtain (14) and the proof is complete.

In a similar way of theorem 3.3 and using lemma 3.4, we can prove the following theorem.

Theorem 3.5. A necessary and sufficient condition for the function $f$ defined by (1) to be in the class of $\mathcal{K}_{n}^{\alpha}[A, B]$ is that

$$
1-\sum_{k=2}^{\infty} \frac{(k-1)\left(e^{i \alpha}+i B \zeta \sin \alpha\right)-(A-k B) \zeta \cos \alpha}{(A-B) \zeta \cos \alpha} k^{n+1} a_{k} z^{k-1} \neq 0
$$

\section{Coefficient Estimates}

In the following, as an applications of Theorems 3.3 and 3.5, we determine coefficient estimates and inclusion properties for a function of the form (1) to be in the classes $\mathcal{S}_{n}^{\alpha}[A, B]$ and $\mathcal{K}_{n}^{\alpha}[A, B]$.

Theorem 4.1. If the function $f(z)$ defined by (1) belongs to $\mathcal{S}_{n}^{\alpha}[A, B]$, then

$$
\sum_{k=2}^{\infty}(|k(B+1)-1|+|\cos \alpha+i B \sin \alpha|) k^{n}\left|a_{k}\right| \leq(A-B) \cos \alpha .
$$


Proof. Since we have

$$
\begin{aligned}
& \left|1-\sum_{k=2}^{\infty} \frac{(k-1)\left(e^{i \alpha}+i B \zeta \sin \alpha\right)-(A-k B) \zeta \cos \alpha}{(A-B) \zeta \cos \alpha} k^{n} a_{k}\right| \\
& \geq 1-\sum_{k=2}^{\infty}\left|\frac{(k-1)\left(e^{i \alpha}+i B \zeta \sin \alpha\right)-(A-k B) \zeta \cos \alpha}{(A-B) \zeta \cos \alpha}\right| k^{n}\left|a_{k}\right|,
\end{aligned}
$$

and

$$
\begin{aligned}
& \left|\frac{(k-1)\left(e^{i \alpha}+i B \zeta \sin \alpha\right)-(A-k B) \zeta \cos \alpha}{(A-B) \zeta \cos \alpha}\right| \\
& =\frac{\left|(k-1)\left(e^{i \alpha}+i B \sin \alpha\right)-(A-k B) \cos \alpha\right|}{(A-B) \cos \alpha} \\
& \leq \frac{|k(B+1)-1|+|A \cos \alpha+i B \sin \alpha|}{(A-B) \cos \alpha},
\end{aligned}
$$

the result follows from Theorem 3.3.

Similarly, we can prove the following theorem.

Theorem 4.2. If the function $f(z)$ defined by (1) belongs to $\mathcal{K}_{n}^{\alpha}[A, B]$, then

$$
\sum_{k=2}^{\infty}(|k(B+1)-1|+|\cos \alpha+i B \sin \alpha|) k^{n+1}\left|a_{k}\right| \leq(A-B) \cos \alpha .
$$

\section{Containment Properties}

In this section, we study the containment properties of the mentioned classes.

Theorem 5.1. $\mathcal{S}_{n+1}^{\alpha}[A, B] \subset \mathcal{S}_{n}^{\alpha}[A, B]$ for all $n \in \mathbb{N}$.

Proof. If $f \in \mathcal{S}_{n+1}^{\alpha}[A, B]$, By the lemma 3.2, we have

$$
\frac{1}{z}\left[f *\left(z+\sum_{k=2}^{\infty}\left(\frac{k-\psi}{1-\psi}\right) k^{n+1} z^{k}\right)\right] \neq 0, \quad z \in \mathbb{U}
$$

where $\psi$ is given by (7). Note that we can write

$$
z+\sum_{k=2}^{\infty}\left(\frac{k-\psi}{1-\psi}\right) k^{n+1} z^{k}=\left(z+\sum_{k=2}^{\infty} k z^{k}\right) *\left(z+\sum_{k=2}^{\infty}\left(\frac{k-\psi}{1-\psi}\right) k^{n} z^{k}\right) .
$$

But

$$
\frac{1}{z}\left[\left(z+\sum_{k=2}^{\infty} k z^{k}\right) *\left(z+\sum_{k=2}^{\infty} k^{-1} z^{k}\right)\right]=1+\sum_{k=2}^{\infty} z^{k-1}=\frac{1}{1-z} \neq 0, \quad z \in \mathbb{U} .
$$

Thus it follows from (17) that

$$
f *\left(z+\sum_{k=2}^{\infty}\left(\frac{k-\psi}{1-\psi}\right) k^{n} z^{k}\right) \neq 0, \quad z \in \mathbb{U}
$$

and we conclude that $f \in \mathcal{S}_{n}^{\alpha}[A, B]$.

Similarly, we can prove the following theorem and corollaries.

Theorem 5.2. $\mathcal{K}_{n+1}^{\alpha}[A, B] \subset \mathcal{K}_{n}^{\alpha}[A, B]$ for all $n \in \mathbb{N}$.

corollary 5.3. $\mathcal{S}_{n}^{\alpha}[A, B] \subset \mathcal{S}^{\alpha}[A, B]$ for all $n \in \mathbb{N}$.

corollary 5.4. $\mathcal{K}_{n}^{\alpha}[A, B] \subset \mathcal{K}^{\alpha}[A, B]$ for all $n \in \mathbb{N}$.

Remark 5.5. In particular, it follow from corollary 5.3 and 5.4 that, $\mathcal{K}^{\alpha}[A, B] \subset$ $\mathcal{S}^{\alpha}[A, B]$. 
Theorem 5.6. If $f \in \mathcal{S}_{n}^{\alpha}[A, B]$ and $\varphi \in \mathcal{K}$, then $f * \varphi \in \mathcal{S}_{n}^{\alpha}[A, B]$ for all $n \in \mathbb{N}$.

Proof. Let.

$$
F:=\frac{z\left(\mathcal{D}^{n} f(z)\right)^{\prime}}{\mathcal{D}^{n} f(z)}
$$

If $f \in \mathcal{S}_{n}^{\alpha}[A, B]$, then $e^{i \alpha} F \prec h$, where $h(z)=\cos \alpha\left(\frac{1+A z}{1+B z}\right)+i \sin \alpha$. Now

$G(z)=\frac{z\left(\varphi * \mathcal{D}^{n} f(z)\right)^{\prime}}{\varphi * \mathcal{D}^{n} f(z)}=\frac{z\left(\varphi *\left(\mathcal{D}^{n} f(z)\right)^{\prime}\right)}{\varphi * \mathcal{D}^{n} f(z)}=\frac{\varphi *\left(z\left(\mathcal{D}^{n} f(z)\right)^{\prime}\right)}{\varphi * \mathcal{D}^{n} f(z)}=\frac{\varphi *\left(F \cdot\left(\mathcal{D}^{n} f(z)\right)\right)}{\varphi * \mathcal{D}^{n} f(z)}$

On the other hand, $f \in \mathcal{S}_{n}^{\alpha}[A, B], \mathcal{D}^{n} f(z) \in \mathcal{S}^{*}$. It follows from Theorem 2.1 [13], that $\frac{\varphi *\left(F \cdot\left(\mathcal{D}^{n} f(z)\right)\right)}{\varphi * \mathcal{D}^{n} f(z)}$ lie in convex hall of $F(\mathbb{U})$. But $e^{i \alpha} F \prec h$ and $h$ is convex, so the convex hall of $e^{i \alpha} F(\mathbb{U})$ is subset of $h(\mathbb{U})$, thus $e^{i \alpha} G(\mathbb{U}) \subset h(\mathbb{U})$, also $e^{i \alpha} G(0)=h(0)$, therefore $e^{i \alpha} G(z) \prec h(z)$ and this completes the proof.

Theorem 5.7. If $f, g \in \mathcal{S}_{n}^{\alpha}[A, B]$. then $f * g \in \mathcal{S}_{n}^{\alpha}[A, B]$ for all $n \geq 1$.

Proof. If $\varphi \in \mathcal{S}_{n}^{\alpha}[A, B]$, then theorem 5.1 provides $\mathcal{S}_{n}^{\alpha}[A, B] \subset \mathcal{S}_{1}^{\alpha}[A, B]=\mathcal{K}_{0}^{\alpha}[A, B]$. On the other hand $\mathcal{K}_{0}^{\alpha}[A, B] \subset \mathcal{K}^{\alpha}[1,-1] \subset \mathcal{K}$. Therefore $\varphi \in \mathcal{K}$. Now by theorem 5.6, $f * \varphi \in \mathcal{S}_{n}^{\alpha}[A, B]$ whenever $f \in \mathcal{S}_{n}^{\alpha}[A, B]$. This completes the proof.

\section{ACKNOWLEDGMEnts}

The Authors would like to express their thanks to Dr. Ahmad Zireh.

\section{REFERENCES}

[1] O. P. Ahuja, Families of analytic functions related to Ruscheweyh derivatives and subordinate to convex functions, Yokohama Math. J. 41 (1993), 39-50.

[2] V. Arora, S. Ponnusami, S.K. Sahoo, Successive coefficients for spirallike and related functions, arXiv:1903.10232 [math.CV] 2019.

[3] S.S. Bhoosnurmath, M.V. Devadas, Subclasses of spirallike functions defined by subordination, J. of Analysis Madras 4, (1996), 173-183.

[4] S.S. Bhoosnurmath, M.V. Devadas, Subclasses of spirallike functions defined by Ruschweyh derivatives, Tamkang J. Math. 28 (1997), 59-65.

[5] J.H Choi, Applications for certain classes of spirallike functions defined by the SrivastavaAttiya operator, APM 8(6), (2018), 615-623.

[6] R. M. Goel and B. S. Mehrok, On the coefficients of a subclass of starlike functions, Indian J. Pure Appl. Math. 12, (1981), 634-647.

[7] A.W.Goodman, Univalent Functions Vol. I Vol. II Polygonal Oublishing House, Washington, FI, (1983).

[8] W. Janowski, Some extremal problems for certain families of analytic functions, Bull. Polish Acad. Sci. 21, (1973), 17-25.

[9] W. Janowski, Some extremal problems for certain families of analytic functions, Annl. Polon. Math. 28, (1973), 297-326.

[10] N. Khan, A. Khan, Q.Z. Ahmad, B. Khan, Study of multivalent spirallike Bazilevic functions, AIMS Mathematics 3(3) (2018), 353-364.

[11] S.V. Nikitin, A class of regular functions, current problems in function theory (Russian) 188, 143-147, Rostov-Gos. Univ. Rostov-on-Don, (1987).

[12] K.I. Noor, Z.H. Bukhari, Some subclasses of analytic and spiral-like functions of complex order involving the SrivastavaAttiya integral operator, INTEGR Trans F SPEC F,1013, 21 (2010), 907-916.

[13] T.N. Shanmugam, Convolution And Differential Subordination, Internat. J. Math. Sci. 12, (1989) 333-340.

[14] H. Silverman and E. M. Silvia, Subclasses of starlike functions subordinate to convex functions, Canad. J. Math. 1, (1985), 48-61.

[15] H. Silverman, Subclasses of starlike functions, Rev. Roumaine. Math. Pures Appl, 231 (1978), 1093-1099.

[16] A. Varsudevararo, FeketeSzegö inequality for certain spiral-like functions, CR MATH. 354(11) (2016), 1065-1070. 